\title{
A zeta potential value determines the aggregate's size of penta-substituted [60] fullerene derivatives in aqueous suspension whereas positive charge is required for toxicity against bacterial cells
}

\author{
Dmitry G. Deryabin ${ }^{1 *}$, Ludmila V. Efremova ${ }^{1,4}$, Alexey S. Vasilchenko ${ }^{1,2}$, Evgeniya V. Saidakova ${ }^{3}$, Elena A. Sizova ${ }^{1,4}$,
} Pavel A. Troshin ${ }^{5}$, Alexander V. Zhilenkov ${ }^{5}$ and Ekaterina A. Khakina ${ }^{5}$

\begin{abstract}
Background: The cause-effect relationships between physicochemical properties of amphiphilic [60]fullerene derivatives and their toxicity against bacterial cells have not yet been clarified. In this study, we report how the differences in the chemical structure of organic addends in 10 originally synthesized penta-substituted [60]fullerene derivatives modulate their zeta potential and aggregate's size in salt-free and salt-added aqueous suspensions as well as how these physicochemical characteristics affect the bioenergetics of freshwater Escherichia coli and marine Photobacterium phosphoreum bacteria. Dynamic light scattering, laser Doppler micro-electrophoresis, agarose gel electrophoresis, atomic force microscopy, and bioluminescence inhibition assay were used to characterize the fullerene aggregation behavior in aqueous solution and their interaction with the bacterial cell surface, following zeta potential changes and toxic effects.

Results: Dynamic light scattering results indicated the formation of self-assembled [60]fullerene aggregates in aqueous suspensions. The measurement of the zeta potential of the particles revealed that they have different surface charges. The relationship between these physicochemical characteristics was presented as an exponential regression that correctly described the dependence of the aggregate's size of penta-substituted [60]fullerene derivatives in saltfree aqueous suspension from zeta potential value. The prevalence of DLVO-related effects was shown in salt-added aqueous suspension that decreased zeta potential values and affected the aggregation of [60]fullerene derivatives expressed differently for individual compounds. A bioluminescence inhibition assay demonstrated that the toxic effect of [60]fullerene derivatives against E. coli cells was strictly determined by their positive zeta potential charge value being weakened against P. phosphoreum cells in an aquatic system of high salinity. Atomic force microscopy data suggested that the activity of positively charged [60]fullerene derivatives against bacterial cells required their direct interaction. The following zeta potential inversion on the bacterial cells surface was observed as an early stage of toxicity mechanism that violates the membrane-associated energetic functions.
\end{abstract}

Conclusions: The novel data about interrelations between physicochemical parameters and toxic properties of amphiphilic [60]fullerene derivatives make possible predicting their behavior in aquatic environment and their activity against bacterial cells.

\footnotetext{
*Correspondence: dgderyabin@yandex.ru

${ }^{1}$ Department of Microbiology, Orenburg State University, Orenburg,

Russia

Full list of author information is available at the end of the article
} 
Keywords: [60]fullerene derivatives, Aqueous suspension, Particle size, Zeta potential, Escherichia coli, Photobacterium phosphoreum, Bioluminescence inhibition assay, Atomic force microscopy, Toxicity

\section{Background}

[60]fullerene ("buckyball") is a hollow spherical molecule exclusively composed of 60 carbon atoms. Thirty years after discovery of the fullerenes [1], they are still a topic of interest for potential application, especially with the advent of nanobiology and nanomedicine [2]. Due to unique characteristics, modified forms of [60]fullerene have been intensively investigated for specific biological activities positioned in various fields, from diagnostics [3, $4]$ to drug delivery $[5,6]$.

A key condition of the biomedical application of fullerenes is good solubility in aqueous media which is necessary for their administration and distribution in the living systems. Because the pristine buckyball is non-polar, the covalent addition of ionic functional groups is a way of making the fullerene derivatives more hydrophilic than the original molecule [7]. If the additive groups are not localized diffusely but concentrated at one pole of the [60]fullerene core, such chemical functionalization leads to amphiphilic compounds called "amphifullerenes" [8]. As a result, these derivatives contain both hydrophobic and hydrophilic moieties and are self-assembled in aqueous environment to spherical aggregates referred to as "buckysomes" [9]. Although the principal methodologies to prepare aqueous suspensions of [60]fullerene derivatives have been developed, the optimal fine structure of additive groups such as final physicochemical characteristics required for effective solubilization are still not clear. According to the PoissonBoltzmann theory, this involves the zeta potential-electric difference in the interfacial double layer around dispersed aggregates that plays an important role in the dispersivity and stability of colloidal solutions [10].

The toxicity of [60]fullerene derivatives for live systems (from bacteria to human beings) is probably triggered by their interactions with cell surface, and therefore different dimensions of fullerene aggregates in aqueous suspensions as well as their physicochemical properties can affect the biological response. In particular, the size, surface chemistry, and surface charge were identified as the properties of [60]fullerene derivatives influencing their toxicity against bacterial cells $[11,12]$. In vitro studies on anionic carboxyfullerene reported its antibacterial activity on Gram-positive species [13], and in vivo it can protect mice from lethal infection of Streptococcus pyogenes [14]. Some aminofullerenes were active against Gram-positive (Enterococcus faecalis) and Gram-negative (Escherichia coli) bacteria [15]. In the latter reports, the novel cationic [60]fullerene derivatives were tested against $E$. coli cells and have shown a potent antibacterial activity comparable to traditional antibiotics. Therefore, some of these compounds can be positioned as new promising agent for disinfection of waters and soil [16] or as potential antibacterial drugs [17]. However, fundamental cause-effect relationships between the physicochemical properties of [60]fullerene derivatives and their affinity to cell surface in order to better predict their toxicity against bacteria have not yet been clarified.

In the present study, we investigated the effects of the chemical structure of organic addends in 10 originally synthesized amphifullerenes on the aggregate's size and zeta potential in aqueous suspensions and revealed important correlations between these physicochemical characteristics and contacts of [60]fullerene derivatives with the bacterial cell surface involved in bioenergetics violation and toxic effect.

\section{Results and discussion}

\section{Characterization of penta-substituted [60]fullerene derivatives in aqueous suspensions}

Ten originally synthesized penta-substituted [60]fullerene derivatives used in this study are presented in Table 1. Each compound consists of a hydrophobic "buckyball" cage and five ionic functional groups attached at one pole, thus including amphiphilic properties and significantly increasing solubility in polar media.

The [60]fullerene derivatives formed red-brown colored aqueous suspensions which were used for hydrodynamic size (diameter) measurement when $10 \mu \mathrm{L}$ aliquots were transferred into the polystyrene cuvettes pre-filled with $1 \mathrm{~mL}$ of deionized water. The following light scattering showed nine out of 10 suspensions as a narrow disperse colloidal systems with one well defined peak including $95.5-100 \%$ of the particles by volume. However, the hydrodynamic size of individual [60]fullerene derivatives varied over the wide range. The smallest average diameter was determined for F9 $(1.9 \pm 0.5 \mathrm{~nm})$ and F10 $(2.5 \pm 0.7 \mathrm{~nm})$ derivatives bearing the residues of the aromatic ring-containing carboxylic acids, while the particles of F5 and F7 derivatives bearing the aminoacid-based addends were somewhat larger $(3.1 \pm 2.0 \mathrm{~nm}$ and $4.6 \pm 0.8$, respectively). These data also showed alkyl chain length in smallest structure of similar additive groups as the cause of decreasing of [60] fullerene derivatives dispersivity: the particle size of F10 compound was more than F9, and the F7 more than F5. The F4 and F8 compounds bearing with diaminebased functional groups showed particles size less than $10 \mathrm{~nm}$ also (Fig. 1a), which, as well as F5, F7, F9, and F10, 


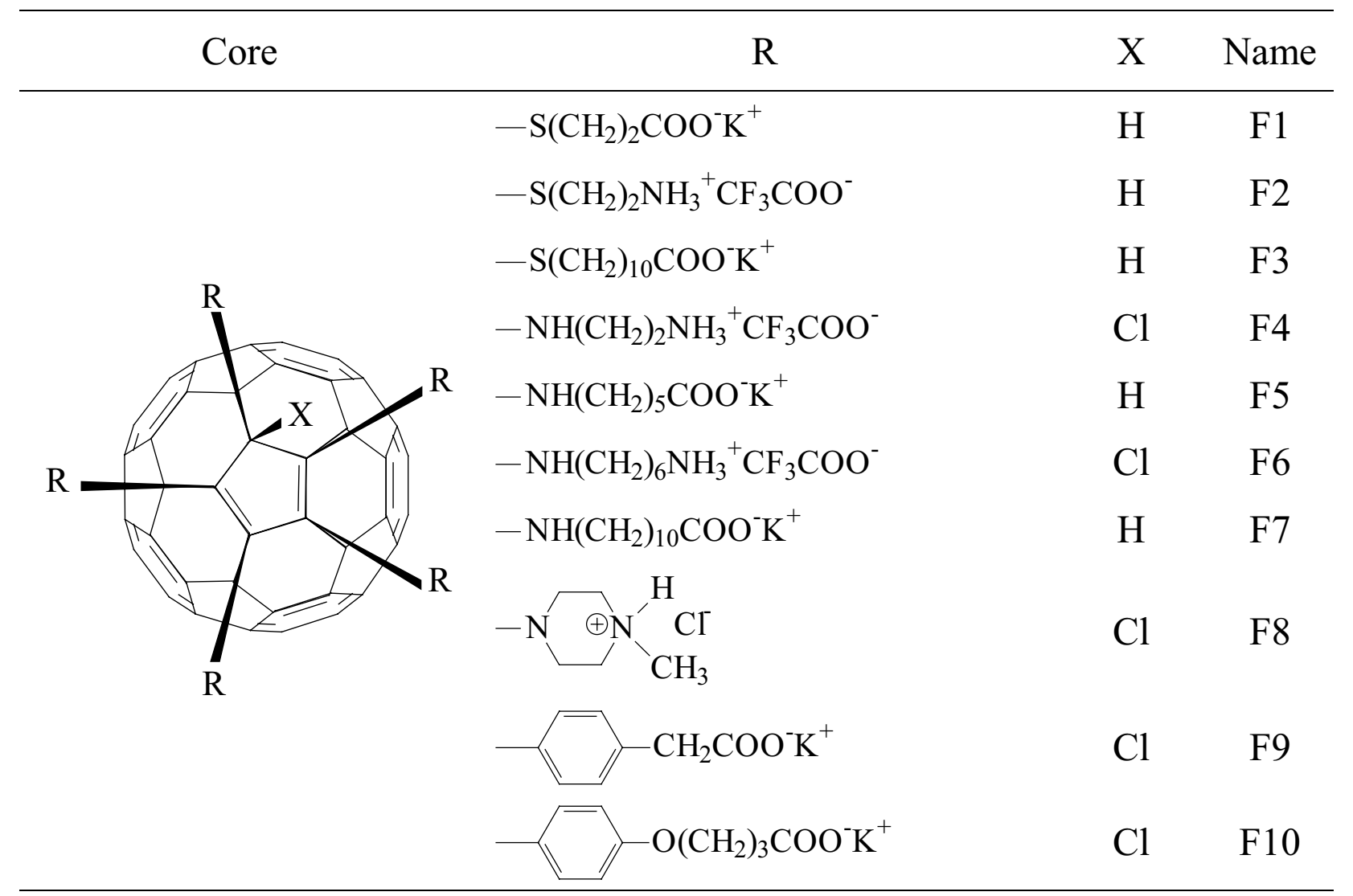
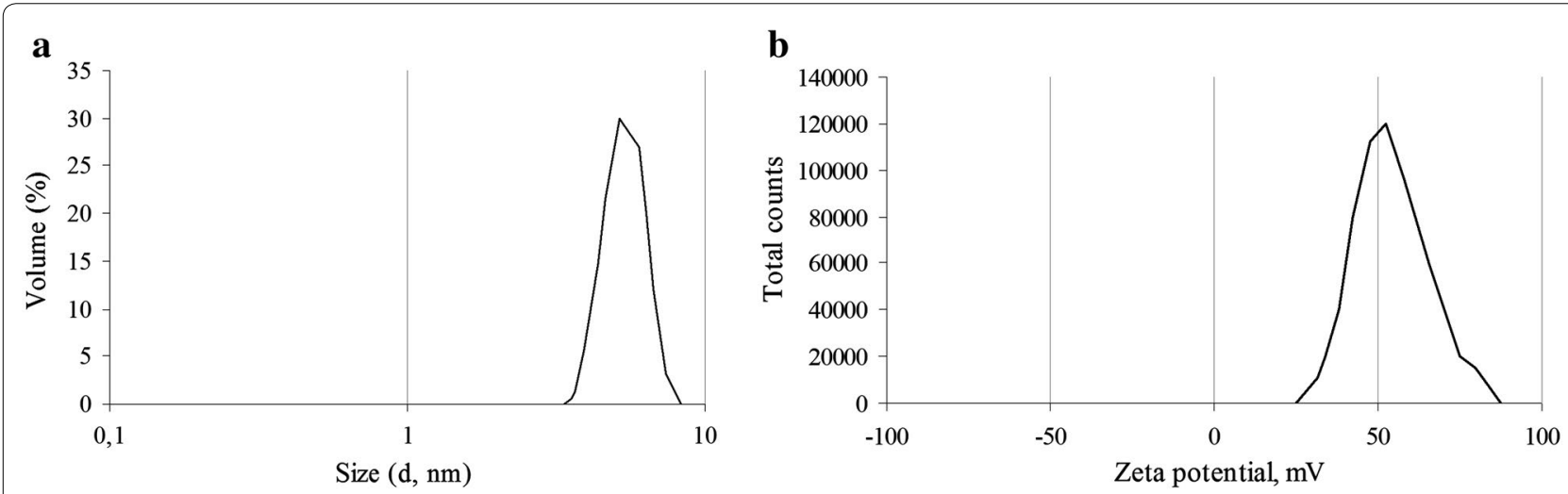

Fig. 1 Example of size and zeta potential distribution in the aqueous suspension of F8 penta-substituted [60]fullerene derivative. a The diameter of F8 aggregates is $5.2 \pm 0.9 \mathrm{~nm}$; b the zeta potential value is $+52.2 \pm 10.6 \mathrm{mV}$.

may represent clusters composed of several fullerene monomers. The hydrodynamic size of F3 particles was established at 10-100 $\mathrm{nm}$ diapasons as $26.6 \pm 3.9 \mathrm{~nm}$ monodisperse aqueous suspension. On the other hand, the average diameter of the aggregates formed by sulfur-containing [60]fullerene derivatives turned out to be more than $100 \mathrm{~nm}$. So, the light scattering data indicated that particles with an average diameter of around
$263.4 \pm 120.0$ and $338.9 \pm 118.7 \mathrm{~nm}$ predominated in F1 and F2 aqueous suspensions, respectively, suggesting the presence of supramolecular aggregates formed by billions of fullerene monomers. Finally, F6 derivative bearing with diamine) addend produced a polydisperse suspension with two maxima at $22.9 \pm 3.0$ and $341.2 \pm 49.2 \mathrm{~nm}$ (65.2 and $34.8 \%$ particles by volume, respectively). 
Atomic force microscopy (data not shown) revealed that particles of [60]fullerene derivatives exhibit spherical shape and confirmed the hydrodynamic size distribution determined by light scattering according to the Smoluchovski equation.

Zeta potential measurements, evaluated by electrophoretic mobility of colloidal particles of [60] fullerene derivatives, indicated that F1, F3, F5, F7, F9, and F10 compounds acquired negative surface charge from $-38.7 \pm 6.5$ to $-57.2 \pm 9.1 \mathrm{mV}$. On the other hand, four compounds show positive surface charge: $+43.4 \pm 6.4 \mathrm{mV}$ for F2, $+49.3 \pm 9.8 \mathrm{mV}$ for F4, $+41.1 \pm 4.5 \mathrm{mV}$ for $\mathrm{F} 6$, and $+52.2 \pm 10.6 \mathrm{mV}$ for $\mathrm{F} 8$ (Fig. 1b), respectively.

\section{Dependence of the particles size in aqueous suspensions of [60]fullerene derivatives on the zeta potential values}

In the current experiment, the functionalization variety, as well as precise determination of particle size and zeta potential values were the basis for analysis of the dependence between these physicochemical characteristics of penta-substituted [60]fullerene derivatives, related to their self-organization in aqueous suspension. From a theoretical viewpoint, the zeta potential values suggest that colloidal particles composed of [60]fullerene derivatives functionalized with ionic groups were surrounded by a stable hydrophilic shell of hydrogenbonded water molecules, which promoted a negative or positive surface charge and prevented the interaction with similar neighboring clusters and aggregates, the zeta potential values were positioned as independent variables $(x, \mathrm{~nm})$, and the particle size values as dependent variables $(y, \mathrm{mV})$.

Figure 2a demonstrates a symmetrical point's distribution against the ordinate axis, while the left and right parts of the graph showed a tendency to decrease in particle size with the increasing surface charge. Assuming that negative or positive electric charge is equally important in colloidal systems and that only the average charge value is significant, the diagram was pre-formed in Fig. $2 \mathrm{~b}$ where the abscissa axis contained the modules of zeta potential values. The following statistical process for estimating the relationships among $(x, y)$ variables gave an exponential regression:

$$
\hat{y}=\exp \left(a_{0}+a_{1} \times x\right),
$$

where coefficient $a_{1}=-0.2789$ and constant $\mathrm{a}_{0}=15.7011$. This model most correctly described the relationships between physicochemical characteristics of penta-substituted [60]fullerene derivatives in water suspension (the accuracy of the mathematical model characterized by F-criterion is 25.2958; $P<0.01$ ). The determination coefficient value for this model,
$R^{2}=0.7193$, led to strict dependence of fullerene aggregation in aqueous suspension from the zeta potential value, determining about three quarters of particle size variability.

The use of the model to predict the clue to zeta potential values required for effective fullerene solubilization and aqueous colloidal suspensions dispersivity yielded two important conclusions. First, this regression showed $|39.8| \mathrm{mV}$ as a critical "border" zeta potential value; exceeding this led to self-assembled fullerene aggregates less than $100 \mathrm{~nm}$ in diameter. The reported satisfactory zeta potential value agrees with the previously published data for colloid [60]fullerenes in water environment $[18,19]$ although there are a few more them, probably because of the non-symmetrical distribution of surface charge in the amphifullerene monomer. Second, the predicted zeta potential value required for single-molecular fullerene aqueous suspension having a hydrodynamic particle size of $1 \mathrm{~nm}$ was $>|56.3| \mathrm{mV}$. However, the fine structure of investigated amphiphilic compounds containing an expressed hydrophobic pole ("buckyball" cage) makes this perspective unlikely, limiting pentasubstituted [60]fullerene derivatives to form clusters composed of more than one fullerene monomer in polar media.

\section{The zeta potential changes and aggregation behavior of [60]fullerene derivatives in salt-added solution}

The environment varies by temperature, $\mathrm{pH}$, ionic strength and dissolved organic matter that alter the behavior of [60]fullerene in natural aquatic systems [20]. According to the classic Derjaguin-Landau-Verwey-Overbeek (DLVO) theory of colloidal stability, the important factor is the presence of electrolytes that varied greatly from freshwater to the marine environment [21]. For this reason, the next step of our experiments was the comparison of [60] fullerene derivative suspensions in salt-free and salt-added media that checked the impact of zeta potential values on aggregation behavior.

Because high electrolyte concentrations limited the laser Doppler micro-electrophoresis, the zeta potential of [60]fullerene derivatives was evaluated by semi-quantitative agarose gel electrophoresis technique [22] with and without $2 \% \mathrm{NaCl}$ in buffer media while the hydrodynamic size of [60]fullerene derivatives in salt-added suspensions was still measured using non-invasive backscatter (NIBS) optical technology.

The electrophoretic mobility of [60]fullerene derivatives in salt-free agarose gel confirmed F1, F3, F5, F7, F9 and F10 compounds as negative surface charged particles and F2, F4, F6 and F8 as positively charged (Fig. 3a). In the presence of $2 \% \mathrm{NaCl}, \mathrm{F} 2$ and $\mathrm{F} 6$ lost their electrophoretic mobility whereas other compounds decreased 

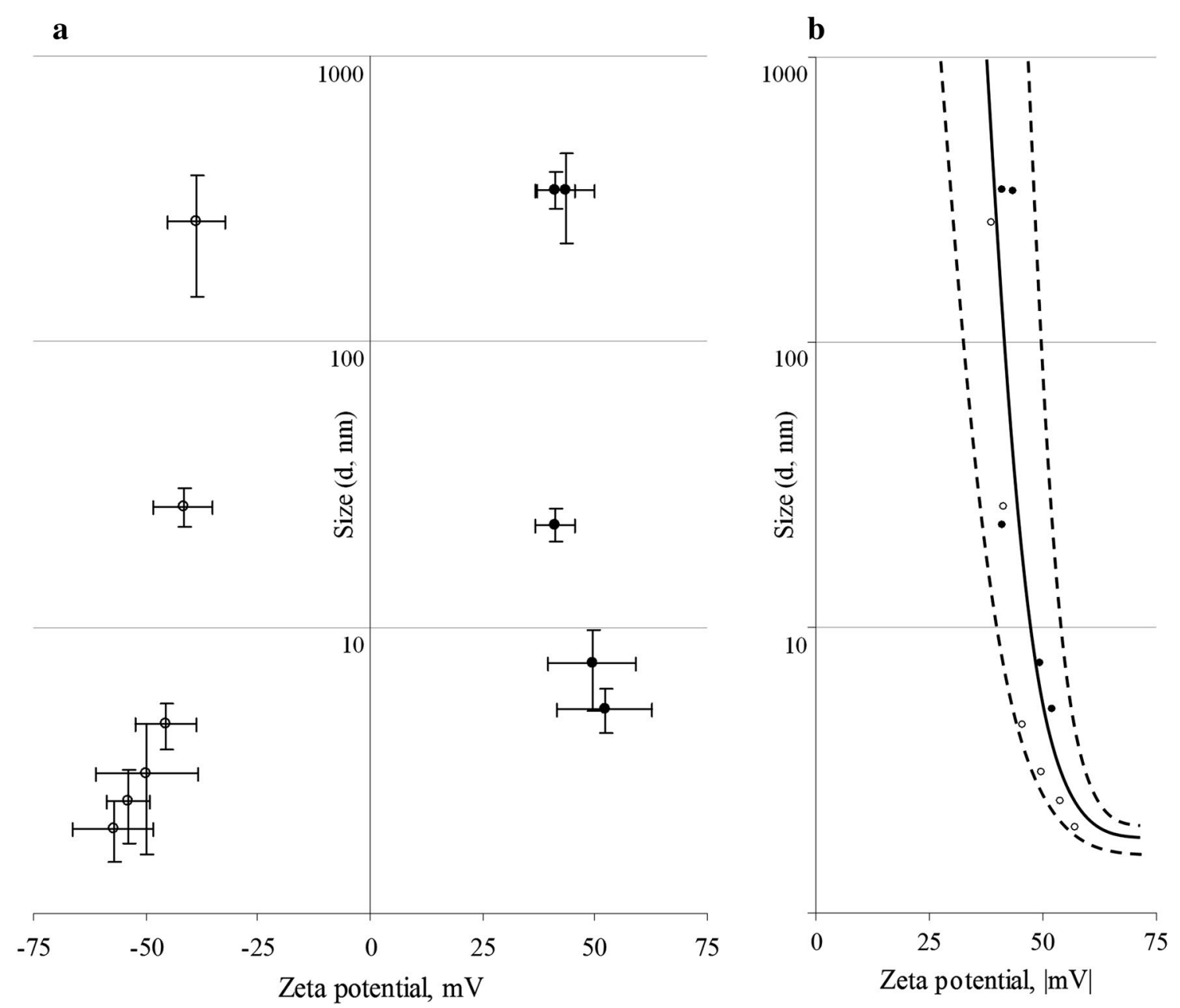

Fig. 2 The graph illustrates distribution of particles size and zeta potential values of [60]fullerene derivatives in aqueous suspension. Data analysis with (a) and without (b) the negative and positive sign of the particles' surface charge. White circles negatively charged compounds; black circles positively charged compounds.

it 1.5-2.5-fold (Fig. 3b), which showed significant neutralization of zeta potential most likely due to opposite charged ions interacting with an electrochemical double layer around the fullerene particles.

A high electrolyte concentration reduced the electrostatic energy barrier between the identically charged nanoparticles, thus leading to an increase in [60]fullerene derivative aggregation expressed differently for individual compounds (Fig. 3c). Being so well dispersed in salt-free media, the F5, F7, F8, F9 and F10 compounds were relatively resistant to electrolyte-induced aggregation while remaining within the range of $10 \mathrm{~nm}$. In turn, F1, F3 and F4 suspensions in the presence of $2 \% \mathrm{NaCl}$ were polidispersive, while F2 and F6 aggregated dramatically, which is evident in the increase in hydrodynamic size (diameter) of 40.7 and $21.3 \%$ of particles over $1000 \mathrm{~nm}$, which in the current experimental context may be interpreted as approaching or exceeding critical coagulation concentrations. On the other hand, in F1 and F8 salt-added suspension we observed partial disaggregation of small particles due to the Debye screening effect [23].

A major implication of this finding is that the behavior of [60]fullerene derivatives in the presence of electrolytes is subjected of several forces distorting the regression dependence as described above. However, the prevalence of the DLVO-type interactions retains the basic dependence of particle size variability from zeta potential value and predicts increased aggregation rates and low bioavailability in aquatic systems of high salinity.

\section{Effect of penta-substituted [60]fullerene derivatives on bacterial bioluminescence}

The toxic effect of [60]fullerene derivatives against freshwater E. coli and marine Photobacterium phosphoreum 


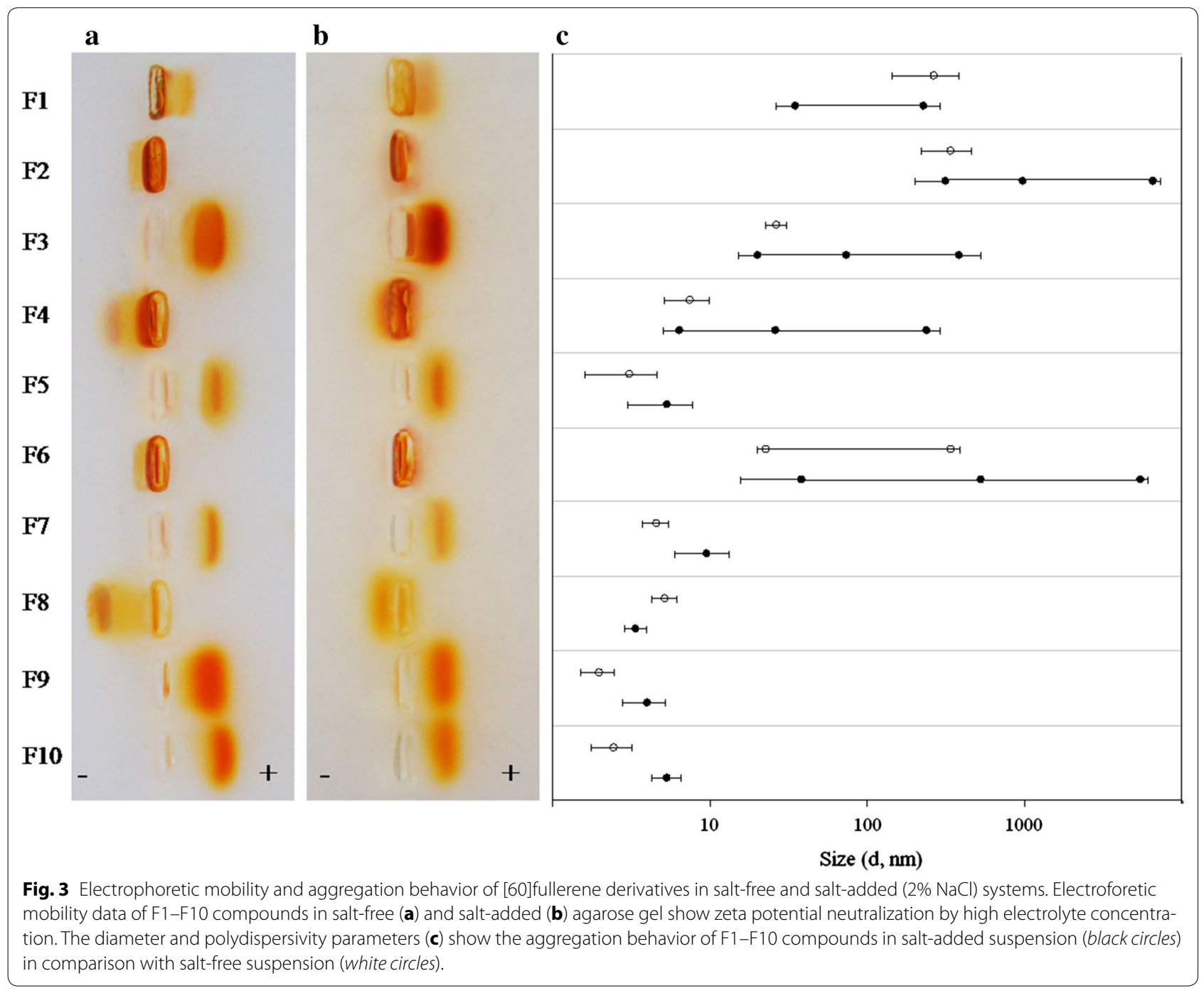

strains was evaluated in salt-free and salt-added media, respectively, by measuring the bacterial bioluminescence level as the direct manifestation of the energetic state of integral cells.

The universal early event of $E$. coli K12 TG1 lac::luxCDABE contact with fullerene suspensions was the rapid light reduction in the first second of instrument registration, which was similar for each [60]fullerene derivative dependent on the compound concentration in the sample. In our experience [24], this effect was not associated with toxicity but was determined by optical distortion of light emission in colored fullerene suspensions. On the other hand, the continued measurement revealed two variants of following bioluminescence kinetics, the first of which had no further changes in light intensity (Fig. 4a), whereas the second show progressivein-time bioluminescence inhibition (Fig. 4b). According to these comparative data the [60]fullerene derivatives were divided into two groups: (1) non-toxic compounds F1, F3, F5, F7, F9, and F10, which did not affect bacterial energetic metabolism; and (2) toxic compounds F2, F4, F6, and F8, which exerted a concentration-dependent and time-dependent bioluminescence inhibition. Based on final indexes the response curves were compiled (Fig. 4c) and the EC50 toxicological parameters were calculated as $94.3 \pm 2.9 \mu \mathrm{mol}$ for F2, $49.5 \pm 1.3 \mu \mathrm{mol}$ for F4, $99.8 \pm 2.7 \mu \mathrm{mol}$ for F6, and $41.2 \pm 1.6 \mu \mathrm{mol}$ for F8, respectively.

The toxicity of [60]fullerene derivatives against the marine bacterium $P$. phosphoreum B17-677F was determined in $2 \% \mathrm{NaCl}$ media. Based on the calculated EC50 value, the ranking order for these compounds is as follows: F8 $(21.9 \pm 0.6 \mu \mathrm{mol})>\mathrm{F} 4(142.7 \pm 5.5 \mu \mathrm{mol})>\mathrm{F} 6$ $(234.0 \pm 9.7 \mu \mathrm{mol})$; whereas the $\mathrm{F} 2$ compound at any of the test concentrations with either of the endpoints became inactive in this biotest. Thus, [60]fullerene 

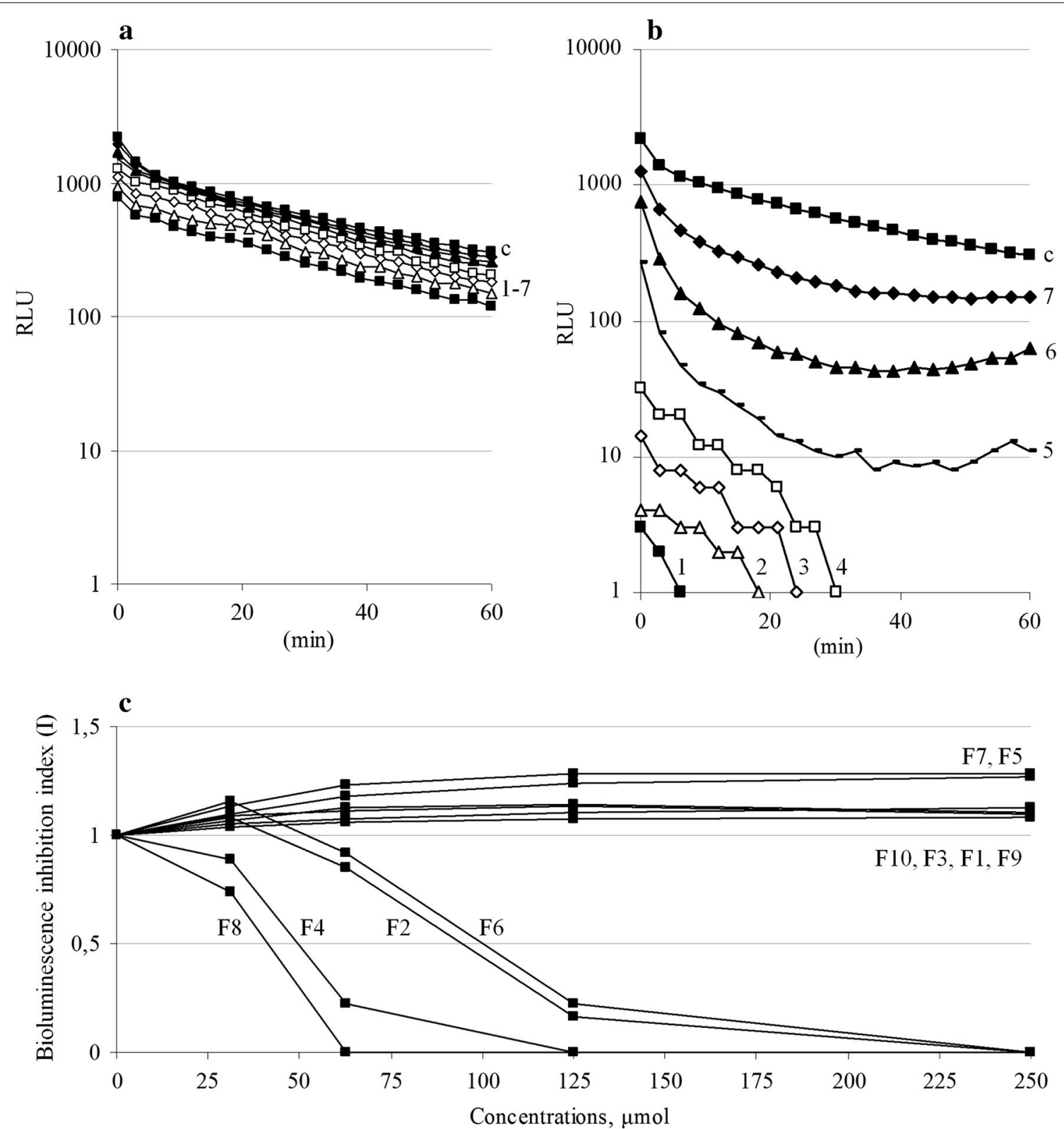

Fig. 4 The toxicity of [60]fullerene derivatives evaluated with bioluminescence inhibition assay. The examples of E. coli K12 TG1 lac::/uxCDABE luminescence time course during contact with aqueous suspensions of F10 (a) and F8 (b) compounds, and the dose-response curves described the changes in the bioluminescence inhibition indexes caused by differing content of penta-substituted [60]fullerene derivatives' (c). Designations $(\mathbf{a}, \mathbf{b})$ : ordinate-bioluminescence, RLU; abscissa—time measurement, min. Compounds concentrations: $12000 \mu \mathrm{mol} ; 21000 \mu \mathrm{mol} ; 3500 \mu \mathrm{mol} ; 4$ $250 \mu \mathrm{mol} ; 5125 \mu \mathrm{mol} ; 662.5 \mu \mathrm{mol} ; 731.25 \mu \mathrm{mol} ;$ c control. Designations (c): ordinate-bioluminescence inhibition index, l; abscissa—[60]fullerene derivatives concentrations, $\mu \mathrm{mol}$.

derivatives, except for the F8 compound, in a salt-added system decreased or lost bioactivity while F1, F3, F5, F7, F9 and F10 were still nontoxic.

Since several penta-substituted [60]fullerene derivatives showed toxic effect against $E$. coli and $P$. phosphoreum cells, the statistical relationship between this bioactivity and fullerenes' physicochemical properties in salt-free and salt-added aqueous suspension was analyzed. The Pearson correlation coefficient for EC50 toxicological parameters and particles size or zeta potential values was calculated, and the results of random variables were shown as the numerical value and linear dependences for sets of data.

Despite the particle size in aqueous suspension being considered significant for pristine [60]fullerene and fullerenol bioactivity $[25,26]$, the role of this parameter 
in penta-substituted [60]fullerene derivatives for $E$. coli K12 TG1 lac::luxCDABE and P. phosphoreum B17$677 \mathrm{~F}$ bioluminescence inhibition was insignificant. The correlation coefficients between these variables were $r=-0.326$ and $r=-0.035(P>0.05)$, respectively, and could not be linearized correctly. On the other hand, the correlation between the zeta potential values of the compared [60]fullerene derivatives and toxicity against $E$. coli was very strong, $r=-0.993(P<0.01)$, and could be presented as a linear relationship between the displayed physicochemical and bioactivity parameters. In turn, the presence of a high electrolyte concentration weakened the toxicity of [60]fullerene derivatives against $P$. phosphoreum; nonetheless, this bioactivity was displayed for positively charged compounds only.

Thus, the principal implication of this result is that [60]fullerene derivative toxicity against bacterial cells is strictly determined by positive surface charge whereas no significant toxicity was found for any negatively charged compounds. In addition, the selective analysis of the group of cationic compounds suggested that their bioactivity variability depends on both particle size and zeta potential values, weakened by the high electrolyte concentrations because of charge neutralization and particle aggregation effects.

\section{AFM evaluation of penta-substituted [60]fullerene derivatives and $E$. coli cells interaction}

Previous studies have shown that pristine [60]fullerene and fullerene derivatives can attach to bacteria and cells $[27,28]$, but it is not clear whether direct contact is necessary for the antibacterial action. For this reason, the next experiments included the AFM microscopy of intact E. coli cells (Fig. 5a) in comparison with the samples treated with non-toxic and toxic penta-substituted [60] fullerene derivatives.

This study did not reveal any bacterial cell surface contacts with negatively charged F1, F3, F5, F7, F9, and F10 compounds, despite various sized round-shaped particles on the mica surface around the cells have being visualized. These features might be attributed to the aggregates of the [60] fullerene derivatives revealed by light scattering measurements (see above). No differences in cell shape, size, or surface roughness were detected when bacterial cells were treated with those compounds which did not exhibit toxic properties (data not shown).

In contrast, the AFM investigation of $E$. coli cells treated with positively charged [60]fullerene derivatives, which exerted a bioluminescence inhibition effect, revealed an affected cell surface structure. The treatment of bacteria with F2 and F6 [60]fullerene derivatives led to covering of bacterial cells with numerous round-shaped particles. For example, treatment of the bacterial cells with F6 led to their covering with multiple aggregates whose sizes varied from 30 to $375 \mathrm{~nm}$ with an average size of $296 \pm 105 \mathrm{~nm}$ (Fig. 5b). On the other hand, in the case of F4 and F8 compounds, the cell surface of the treated bacteria was covered by finely dispersed particles (Fig. 5c, d, respectively) with an average diameter less than 10-20 $\mathrm{nm}$.

In the current experimental context, it is important that the size of the individual round-shaped particles revealed by AFM corresponds well to the light scattering data (see above), thus proving that these clusters represent self-assembled fullerene aggregates interacting with the bacterial cell surface. Other morphological characteristics (length, width, and height) of E. coli cells were weak changed only except for F2 compound, where the cell's morphology changed more significantly. However, no evidences for appearance of grooves or lesions of the outer membrane or intracellular content efflux were observed thus suggesting that membrane disruption can hardly be considered as a leading mechanism of the antibacterial action of the fullerene derivatives [12, 29].

The obtained results demonstrate that the activity of the positively charged penta-substituted [60]fullerene derivatives on $E$. coli cells requires their direct interaction at the initial stage which might affect also bacterial energetic state.

\section{Effect of penta-substituted [60]fullerene derivatives on zeta potential of Escherichia coli cells}

We have shown that only the positively charged [60] fullerene derivatives interact with the bacterial cell surface. Therefore, we assumed that electrostatic (Colomb) attraction plays a major role in the antibacterial action of these fullerene derivatives. The experimental zeta potential of E. coli K12 TG1 lac::luxCDABE cells measured in an aqueous suspension (Fig. 6) corresponds to the surface potential of $-40.9 \pm 5.8 \mathrm{mV}$, that fits well to the previously reported data on the charge state of the outer surface of the pro- and eukaryotic cells [30].

The effect of toxic concentrations for penta-substituted [60] fullerene derivatives on the zeta potential of $E$. coli cells was evaluated by laser Doppler micro-electrophoresis when $10 \mu \mathrm{L}$ aliquots of previously prepared bacteria and fullerene mixtures were added to a cuvette filled with $1 \mathrm{ml}$ of deionized water. It has been shown that introduction of the cationic fullerene derivatives results in significant changes of the zeta potential of $E$. coli cells. Gradually increasing concentrations of fullerenes led to inversion of the surface charge (Fig. 6). For the same concentration of positively charged [60]fullerene derivatives, the effects exerted by F8 and F4 were more severe than those exerted by F2 and F6. The zeta potential of the 

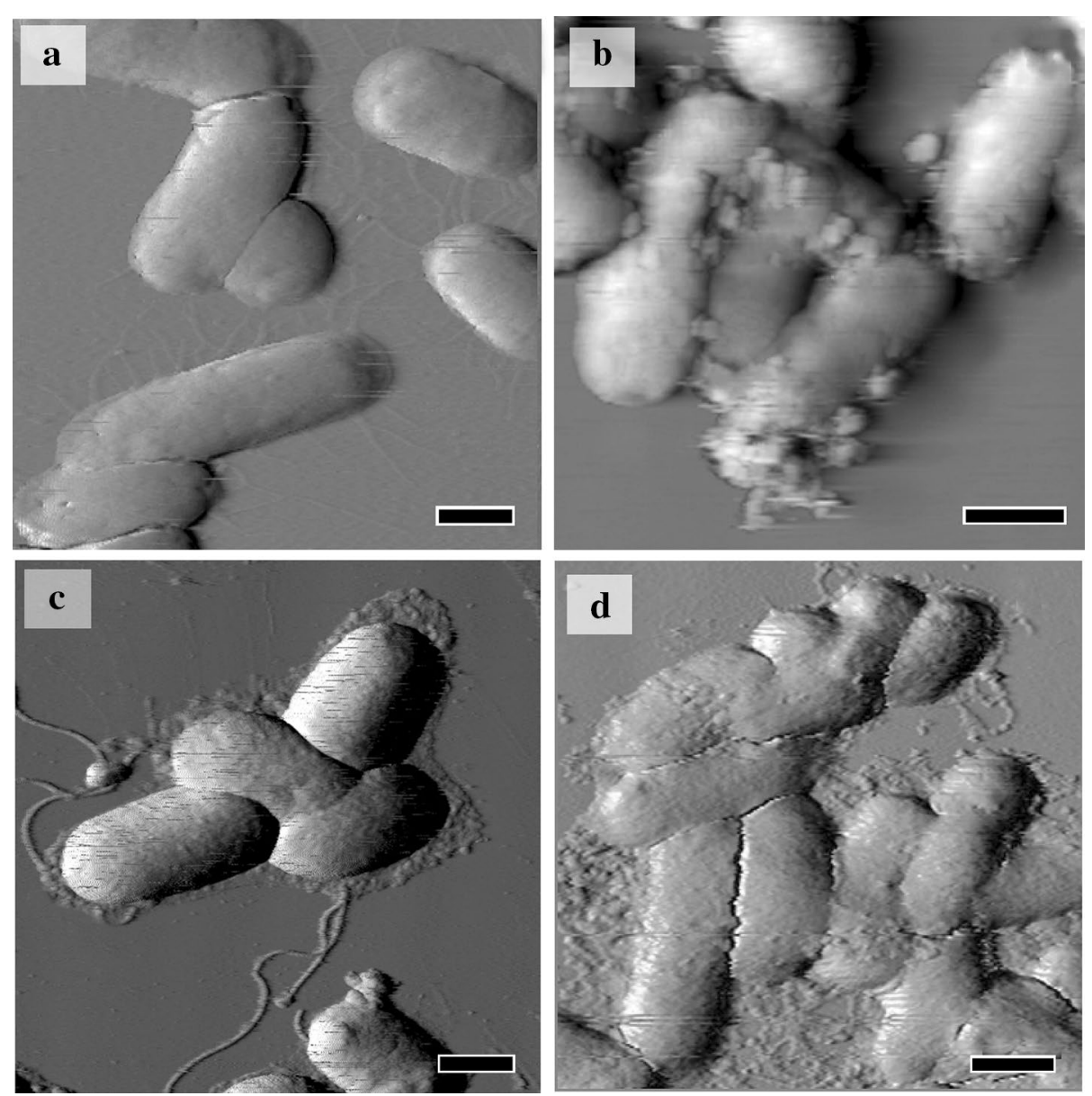

Fig. 5 Contact of positively charged [60]fullerene derivatives with bacterial cell surface evaluated with AFM technique. AFM images of Escherichia coli cells in control sample (a) and treated with F6 (b), F4 (c) and F8 (d) penta-substituted [60]fullerene derivatives. The scale bar $1 \mu \mathrm{m}$.

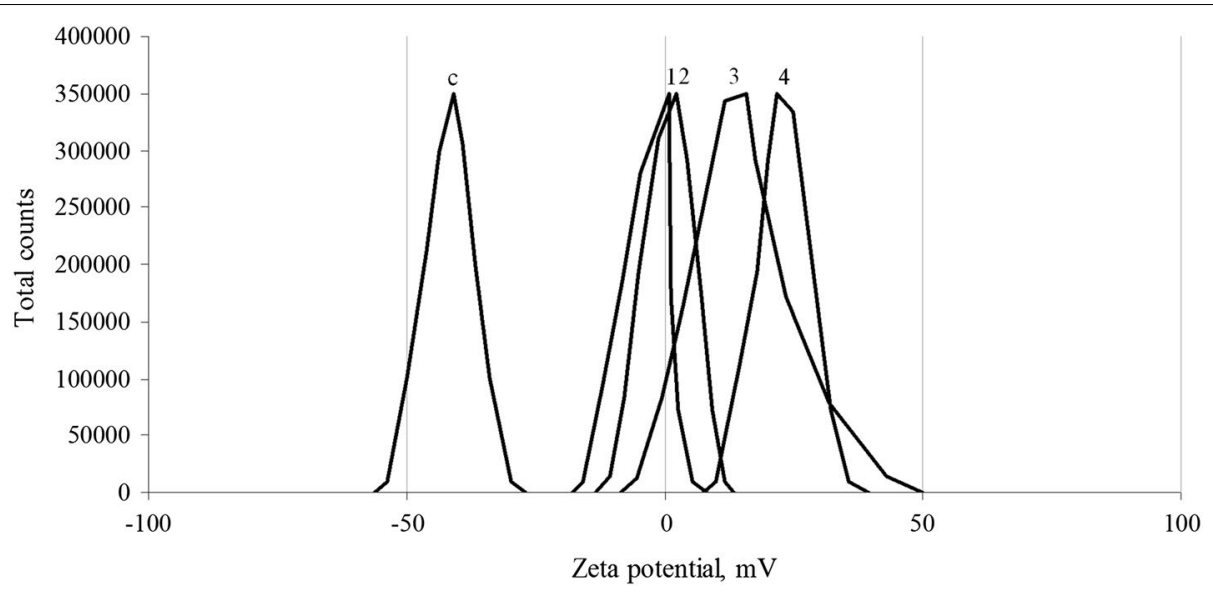

Fig. 6 Influence of positively charged [60]fullerene derivatives on zeta potential of Escherichia coli K12 TG1 cells. The zeta potential distribution for intact (c) and treated with toxic concentrations of F6 (1), F2 (2), F8 (3), and F4 (4) bacterial suspensions. 
bacterial cells changed from $-40.9 \pm 5.8 \mathrm{mV}$ in the control sample to $+15.9 \pm 10.4 \mathrm{mV}(\mathrm{F} 8)$ and $+21.7 \pm 4.9 \mathrm{mV}$ (F4) against $+2.3 \pm 4.5 \mathrm{mV}$ (F2) and $+0.7 \pm 3.8 \mathrm{mV}$ (F6). These results are in a good agreement with the data on the antibacterial action of the fullerene derivatives studied using bioluminescence inhibition assay (see above).

In the current experimental context, this inversion was determined by coverage of bacterial surface with positively charged fullerenes. According to the AFM data, the aggregates of the cationic fullerene derivatives had strong Coulombic attraction to the negatively charged $E$. coli cells surface, whereas negatively charged compounds cannot interact effectively with bacteria due to the Coulombic repulsion. Another interesting fact is that comparable concentrations of $\mathrm{F} 4$ and $\mathrm{F} 8$ compounds with high surface charge and represented by small clusters composed of several fullerene monomers led to more pronounced zeta potential inversion. Most likely, it is due to a complete bacterial surface coverage, while the F2 and F6 aggregates induced less pronounced effect because of the point interaction with the bacterial cell surface.

These results indicate that direct contact of positively charged penta-substituted [60]fullerene derivatives with E. coli cells surface is governed mostly by the electrostatic forces, while the following zeta potential inversion may be an early stage of the toxicity mechanism that probably also involves membrane-associated energetic and transport functions [27, 31].

\section{Conclusions}

In this study, we identified the interrelations between basic physicochemical characteristics of 10 originally synthesized penta-substituted [60]fullerene derivatives with amphiphilic properties and their behavior in aqueous suspensions that is significant for toxic effect against freshwater E. coli and marine P. phosphoreum cells. The obtained results confirmed the Poisson-Boltzmann theory for self-assembly of amphifullerene suspensions. A clear relationship between the zeta potentials of the functionalized [60]fullerene aggregates and their size in salt-free aqueous media has been revealed for the first time. We have also demonstrated a prevalence of Derjaguin-Landau-Verwey-Overbeek-related effects in salt-added aqueous media that decreased zeta potential and affected the aggregation behavior of tested compounds. Notably, the positive surface charge of the fullerene nanoparticles governs their interactions with the negatively charged $E$. coli cells' surface in a salt-free system and, consequently, their effect on the bacterial bioenergetics, as confirmed by the strong bioluminescence inhibition effect. In turn, the [60]fullerene derivatives' toxicity against $P$. phosphoreum cells is weakened due to salt-induced zeta potential neutralization and aggregation effects. It has been shown that direct interaction of positively charged [60]fullerene aggregates with bacterial surface and following inversion of the zeta potential of bacterial cells occurs at an early stage of the toxicity mechanism. These results provide novel insights to unveil the molecular mechanisms underlying amphyfullerene biological activity so demonstrating a promising strategy for designing effective antibacterial preparations based on penta-substituted [60]fullerene derivatives.

\section{Methods}

\section{[60]fullerene derivatives: synthetic procedures}

\section{and suspension preparation}

Ten penta-substituted [60]fullerene derivatives were synthesized according to the previously published highly selective reactions $[32,33]$ starting from readily available chlorofullerene $\mathrm{C}_{60} \mathrm{Cl}_{6}$ as a precursor. These reactions open up straightforward synthetic routes to many functionalized fullerene derivatives with high yields, for example water-soluble compounds showing various biological activities.

Aqueous suspensions of [60]fullerene derivatives $(4 \mathrm{mmol})$ were prepared in deionized water or $2 \% \mathrm{NaCl}$ solution in glass vials, vigorously vortexed and sonicated $\left(30 \mathrm{~W} \times \mathrm{dm}^{-3}, 35 \mathrm{kHz}\right)$ for $30 \mathrm{~min}$ in a water bath. The suspensions were then incubated for about $2 \mathrm{~h}$ at $20^{\circ} \mathrm{C}$, thus allowing the colloidal systems to reach equilibrium.

\section{Bacterial strains and culture preparation}

An E. coli $\mathrm{K} 12$ TG1 host-based recombinant strain commercially available as "Ekolum" biosensor (Immunotekh, Russia) was the test object for toxicological screening. This strain carries a pF1 plasmid with the luxCDABE operon of marine bioluminescent bacteria Vibrio fischeri cloned under the lac promoter that gives strong constitutive light emission under standard cultivation conditions. Another sensor strain was the marine bacterium $P$. phosphoreum commercially available as "Microbiosensor B17-677F" [34] carrying the natural lux operon in a bacterial chromosome. For both strains the inhibition of bioluminescence is likely due to the toxicity associated with reducing bacterial energetics, because light production requires active NADH and ATP metabolism.

According to the manufacturer's recommendation, the E. coli K12 TG1 lac::luxCDABE lyophilized sensor strain was rehydrated with cooled deionized water and $P$. phosphoreum B17-677F with cooled $2 \% \mathrm{NaCl}$ solution to a concentration about $4 \times 10^{8}$ colony-forming units per $1 \mathrm{~mL}$, exposed for $30 \mathrm{~min}$ at $2-4^{\circ} \mathrm{C}$ and then the temperature was raised to $20 \pm 2^{\circ} \mathrm{C}$ before use, respectively. 


\section{Measurement of the size particles and zeta potential in aqueous suspensions}

The size and zeta potential of penta-substituted [60] fullerene derivatives dispersed in salt-free aqueous suspensions were assessed with a laser autocorrelation analyzer, Zetasizer Nano (Malvern Instruments Ltd, United Kingdom).

The basic principle of particle size measurement was a non-invasive back scatter (NIBS) optical technology that estimates real-time changes in the intensity of scattered light as a result of particles undergoing Brownian motion. The samples were placed in $10 \times 10 \times 45 \mathrm{~mm}$ polystyrene cuvettes with four optical faces and optimal transparency along the spectral field from 340$800 \mathrm{~nm}$ (Kartell Labware, Italy), and were illuminated by a $633 \mathrm{~nm}$ helium-neon laser. The light scattering was measured at an angle of $173^{\circ}$ using an avalanche photodiode, and the particles' size distribution was calculated from the diffusion coefficient according to the Smoluchovski equation, which is known to be rigorously valid for spherical-like particles. The average diameter \pm width $(\mathrm{nm})$ of the aggregates of [60]fullerene derivatives in aqueous suspensions was calculated according to the volume size distribution data by using the software of the instrument.

The zeta potential of [60]fullerene derivatives, bacterial cells, and complexes thereof were measured using the technique of laser Doppler micro-electrophoresis. In this method, an electric field is applied across a pair of electrodes to the particle dispersion, which then move with a velocity related to its surface charge values. This parameter is measured using a laser phase analysis light scattering technique (M3-PALS) that enables the calculation of electrophoretic mobility and performs it to mean \pm width $(\mathrm{mV})$ zeta potential and zeta potential distribution.

The aggregation behavior of penta-substituted [60] fullerene derivatives dispersed in salt-added aqueous suspensions was analyzed with NIBS optical technology while zeta potential was evaluated with $1 \%$ agarose gel electrophoresis with and without $2 \% \mathrm{NaCl}$ in $0.1 \%$ phosphate buffer media ( $\mathrm{pH}$ 7.2). Electrophoresis was performed in parallel connected chambers at constant voltage of $20 \mathrm{~V}$ and current of $200 \mathrm{~mA}$, so that the electric field strength was about $0.7 \mathrm{~V} / \mathrm{cm}$. After $30 \mathrm{~min}$ of electrophoresis, migration of compounds was evaluated by visible light and the Smoluchowski equation was used to calculate the zeta potential from the electrophoretic mobility.

\section{Atomic force microscopy}

Visualization of investigated compounds, bacterial cells, and contacts between them was performed using an atomic force microscope SMM-2000 (Proton-MIET,
Russia) as described previously [35]. Briefly, aliquots $(20 \mu \mathrm{L})$ of aqueous suspensions of [60]fullerene derivatives, E. coli K12 TG1 cells, alone or previously mixed with fullerene suspensions, were applied to freshly prepared mica. The samples were incubated at $93 \%$ relative humidity and $20-22^{\circ} \mathrm{C}$ and scanned in a contact mode using V-shaped silicon nitride cantilevers MSCT-AUNM (Veeco Instruments Inc., USA) with a spring constant of $0.01 \mathrm{~N} / \mathrm{m}$ and a tip curvature of $10 \mathrm{~nm}$. Quantitative morphometric analysis of the images was performed using the software provided with the instrument.

\section{Bioluminescent toxicological assay}

To assess toxicity of [60]fullerene derivatives against E. coli K12 TG1 lac::luxCDABE and P. phosphoreum B17-677F cells we used a previously described version of bioluminescent analysis for carbon-based nanomaterials [20]. Briefly, aqueous suspensions of [60]fullerene derivatives $(4 \mathrm{mmol})$ were added to the wells of a "Microlite 2+" microplate with non-transparent side walls (Thermo, USA), wherein they were further doubly diluted in sterile deionized water or $2 \% \mathrm{NaCl}$ solution, from $1: 1$ to $1: 1,024$, up to a final volume of $50 \mu \mathrm{L}$. Then, $50 \mu \mathrm{L}$ of a previously prepared suspension of constitutively luminescent $E$. coli or $P$. phosphoreum cells was added to the wells filled with salt-free or salt-added suspensions, respectively. Wells filled with sterile deionized water or $2 \% \mathrm{NaCl}$ solution and containing an appropriate amount of bacterial biosensor were used as controls.

Bioluminescence measurements were carried out using an LM-01T microplate luminometer (Immunotech, Czech Republic), which dynamically registered the luminescence intensity of the samples for $60 \mathrm{~min}$, estimated in relative light units (RLU). The data were analyzed using KILIA graphing software provided with the instrument. To quantify the bioluminescence inhibition index (I) due to toxicity of [60]fullerene derivatives we used the algorithm I $=\mathrm{RLU} c 0 \times \mathrm{RLUtn} / \mathrm{RLU} c n \times \mathrm{RLU} t 0$, where $c$ and $t$ are the RLU values of the control and test samples at the 0 -th and $n$-th minute of measurement. Based on these indexes, we calculated the EC50 toxicological parameters, that is, the concentrations of [60]fullerene derivatives that cause $50 \%$ bioluminescence inhibition.

\section{Statistical analysis}

All the experiments were performed using three or more independent series with different preparations. The values were expressed as mean \pm standard deviation. The regression analysis for estimating the relationships among zeta potential and particle size values was processed using the software package Statistica V8 (StatSoft Inc., United States). Statistical significance was set at $p<0.05$. 


\section{Abbreviations}

AFM: atomic force microscopy; NIBS: non-invasive back scatter; RLU: relative light units.

\section{Authors' contributions}

DGD supervised the study, and contributed to the selection of methodology, analysis of the obtained results, and drafting of the manuscript. LVE executed the experimental studies on bioluminescent assay. ASV performed the atomic force microscopy. EVS helped to perform the measurement of the size particles and zeta potential. EAS performed the statistical analysis of the data. PAT, $A V Z$ and EAK worked on the synthesis of the water-soluble penta-substituted [60]fullerenes. All authors read and approved the final manuscript.

\section{Author details}

${ }^{1}$ Department of Microbiology, Orenburg State University, Orenburg, Russia.

${ }^{2}$ Institute of Cellular and Intracellular Symbiosis, RAS, Orenburg, Russia.

${ }^{3}$ Institute for Ecology and Genetics of Microorganisms, RAS, Perm, Russia.

${ }^{4}$ All-Russia Research Institute of Beef Cattle Breeding, RAS, Orenburg, Russia.

${ }^{5}$ Institute for Problems of Chemical Physics of RAS, Chernogolovka, Russia.

\section{Acknowledgements}

The physicochemical characterization of [60]fullerene derivatives in aqueous suspensions was supported by Russian Foundation for Basic Research (project no. 15-04-04379_a). Toxicological experiments were performed according the project no. 14-36-00023 from the Russian Science Foundation. Synthesis of penta-substituted [60]fullerenes was supported by Russian Foundation for Basic Research (project no. 14-03-32058) and Russian President Science Foundation (MK-7166.2015.3). We thank Dr. A. B. Kornev for participation in the synthesis of some compounds and A. M. Korotkova for agarose gel electrophoresis assistance.

\section{Compliance with ethical guidelines}

\section{Competing interests}

The authors declare that they have no competing interests.

Received: 7 April 2015 Accepted: 29 July 2015

Published online: 08 August 2015

\section{References}

1. Kroto HW, Heath JR, O'Brien SC, Curl RF, Smalley RE (1985) C60: buckminsterfullerene. Nature 318:162-163

2. Bakry R, Vallant RM, Najam-ul-Haq M, Rainer M, Szabo Z, Huck CW et al (2007) Medicinal applications of fullerenes. Int J Nanomedicine 2(4):639-649

3. Chen Z, Mao R, Liu Y (2012) Fullerenes for cancer diagnosis and therapy: preparation, biological and clinical perspectives. Curr Drug Metab 13(8):1035-1045

4. Dellinger A, Zhou Z, Connor J, Madhankumar AB, Pamujula S, Sayes CM et al (2013) Application of fullerenes in nanomedicine: an update. Nanomedicine (Lond) 8(7):1191-1208

5. Bolskar RD (2012) Fullerenes for drug delivery. In: Bhushan B (ed) Encyclopedia of Nanotechnology. Springer, Netherlands, pp 898-911

6. Lin CM, LU TY (2012) C60 fullerene derivatized nanoparticles and their application to therapeutics. Recent Pat Nanotechnol 6(2):105-113

7. Richardson CF, Schuster DI, Wilson SR (2000) Synthesis and characterization of water-soluble amino fullerene derivatives. Org Lett 2(8):1011-1014

8. Burghardt S, Hirsch A, Schade B, Ludwig K, Bottcher C (2005) Switchable supramolecular organization of structurally defined micelles based on an amphiphilic fullerene. Angew Chem Int Ed Engl 44(19):2976-2979

9. Partha R, Lackey M, Hirsch A, Casscells SW, Conyers JL (2007) Self assembly of amphiphilic $\mathrm{C}_{60}$ fullerene derivatives into nanoscale supramolecular structures. J Nanobiotechnology 5:6. doi:10.1186/1477-3155-5-6

10. Delgado AV, Gonzalez-Caballero F, Hunter RJ, Koopal LK, Lyklema J (2007) Measurement and interpretation of electrokinetic phenomena. J Colloid Interface Sci 309(2):194-224
11. Sayes CM, Fortner JD, Guo W, Lyon D, Boyd AM, Ausman KD et al (2004) The differential cytotoxicity of water-soluble fullerenes. Nano Lett 4(10):1881-1887

12. Tang YJ, Ashcroft JM, Chen D, Min G, Kim CH, Murkhejee B et al (2007) Charge-associated effects of fullerene derivatives on microbial structural integrity and central metabolism. Nano Lett 7(3):754-760

13. Tsao N, Luh TY, Chou CK, Chang TY, Wu JJ, Liu CC et al (2002) In vitro action of carboxyfullerene. J Antimicrob Chemother 49(4):641-649

14. Tsao N, Luh TY, Chou CK, Wu JJ, Lin YS, Lei HY (2001) Inhibition of group A streptococcus infection by carboxyfullerene. Antimicrob Agents Chemother 45(6):1788-1793

15. Mashino T, Nishikawa D, Takahashi K, Usui N, Yamori T, Seki M et al (2003) Antibacterial and antiproliferative activity of cationic fullerene derivatives. Bioorg Med Chem Lett 13(24):4395-4397

16. Deryabin D, Davydova O, Yankina Z, Vasilchenko A, Miroshnikov S, Kornev A et al (2014) The activity of [60]fullerene derivatives bearing amine and carboxylic solubilizing groups against Escherichia coli: a comparative study. J Nanomaterials. doi:10.1155/2014/907435

17. Patel MB, Harikrishnan U, Valand NN, Modi NR, Menon SK (2013) Novel cationic quinazolin-4(3H)-one conjugated fullerene nanoparticles as antimycobacterial and antimicrobial agents. Arch Pharm (Weinheim) 346(3):210-220

18. Deguchi S, Alargova RG, Tsujii K (2001) Stable dispersions of fullerenes, C60 and C70, in water. Preparation and characterization. Langmuir 17(19):6013-6017

19. Wierzbicki M, Sawosz E, Grodzik M, Prasek M, Jaworski S, Chwalibog A (2013) Comparison of anti-angiogenic properties of pristine carbon nanoparticles. Nanoscale Res Lett 8(1):195

20. Yang Y, Nakada N, Nakajima R, Yasojima M, Wang C, Tanaka H (2013) $\mathrm{pH}$, ionic strength and dissolved organic matter alter aggregation of fullerene C60 nanoparticles suspensions in wastewater. J Hazard Mater 244-245:582-587

21. Chen $\mathrm{KL}$, Elimelech M (2006) Aggregation and deposition kinetics of fullerene (C60) nanoparticles. Langmuir 22(26):10994-11001

22. XiaoWei M, ZhiJun C, YanHua L, TianYu G, Xing-Jie L (2010) Application of electrophoresis in the separation of nanoparticles. Scientia Sin Chim 40(10):1478-1486

23. Su-Zhen H, Holger M, Chen-Xu W (2014) Aggregation of fullerene $\left(C_{60}\right)$ nanoparticle: a molecular-dynamic study. Chin Phys B 23(4):048201-048204

24. Deryabin DG, Aleshina ES, Efremova LV (2012) Application of the inhibition of bacterial bioluminescence test for assessment of toxicity carbonbased nanomaterials. Microbiology 81(4):492-497

25. Chae SR, Badireddy AR, Farner Budarz J, Lin S, Xiao Y, Therezien M et al (2010) Heterogeneities in fullerene nanoparticle aggregates affecting reactivity, bioactivity, and transport. ACS Nano 4(9):5011-5018

26. Lyon DY, Adams LK, Falkner JC, Alvarezt PJ (2006) Antibacterial activity of fullerene water suspensions: effects of preparation method and particle size. Environ Sci Technol 40(14):4360-4366

27. Lyon DY, Alvarez PJJ (2008) Fullerene water suspension ( $\mathrm{nC}_{60}$ ) exerts antibacterial effects via ROS-independent protein oxidation. Environ Sci Technol 42(21):8127-8132

28. Su Y, Xu JY, Shen P, Li J, Wang L, Li Q et al (2010) Cellular uptake and cytotoxic evaluation of fullerenol in different cell lines. Toxicology 269(2-3):155-159

29. Wang C, Wang L, Wang Y (2012) Toxicity effects of four typical nanomaterials on the growth of Escherichia coli, Bacillus subtilis and Agrobacterium tumefaciens. Environ Earth Sci 65(6):1643-1649

30. Kłodzinska E, Szumski M, Dziubakiewicz E, Hrynkiewicz K, Skwarek E, Janusz W et al (2010) Effect of zeta potential value on bacterial behavior during electrophoretic separation. Electrophoresis 31(9):1590-1596

31. Mashino T, Okuda K, Hirota T, Hirobe M, Nagano T, Mochizuki M (1999) Inhibition of E. coli growth by fullerene derivatives and inhibition mechanism. Bioorg Med Chem Lett 9(20):2959-2962

32. Kornev AB, Khakina EA, Martynenko VM, Troshin PA, Troyanov SI, Kushch AA et al (2012) Facile preparation of amine and amino acid adducts of [60]fullerene using chlorofullerene of $\mathrm{C}_{60} \mathrm{Cl}_{6}$ as a precursor. Chem Commun 48(44):5461-5463

33. Khakina EA, Yurkova AA, Mumyatov AV, Martynenko VM, Troshin PA, Peregudov AS et al (2012) Highly selective reactions of $\mathrm{C}_{60} \mathrm{Cl}_{6}$ with thiols 
for the synthesis of functionalized [60]fullerene derivatives. Chem Commun (Camb) 48(57):7158-7160

34. Vydryakova GA, Chugaeva Yu V, Tyulkova NA, Medvedeva SE, Kuznetsov AM et al (2005) Luminous bacteria: biotechnological aspects. In: Tsuji A, Matsumoto M, Maeda M, Krika LJ, Stanley Ph. E (eds) Biolum\&Chemilum: Progress \& Perspectives. World Scientific Publishing, New Jersey pp. 95-98
35. Deryabin DG, Vasilchenko AS, Aleshina ES, Tlyagulova AS, Nikiyan AN (2010) An investigation into the interaction between carbon-based nanomaterials and Escherichia coli cells using atomic force microscopy. Nanotechnol Russ 5(11-12):857-863

Submit your next manuscript to BioMed Central and take full advantage of:

- Convenient online submission

- Thorough peer review

- No space constraints or color figure charges

- Immediate publication on acceptance

- Inclusion in PubMed, CAS, Scopus and Google Scholar

- Research which is freely available for redistribution

Submit your manuscript at

www.biomedcentral.com/submit

() BioMed Central 